\title{
Measurement and analysis of fine particulate matters (PM10/PM2.5) and condensable nanoparticles emission from stationary sources
}

\author{
H. Kamiya ${ }^{1}$, K. Hada $^{1}$, T. Sekizawa ${ }^{1}$, M. Yamada ${ }^{1}$, M. Tsukada ${ }^{1}$, \\ W. Lenggoro ${ }^{1}$, M. Wada ${ }^{2}$, N. Kogure ${ }^{3}$, Y. Yuping ${ }^{4}$ \\ \& W. W. Szymanski ${ }^{5}$ \\ ${ }^{1}$ Intitute of Engineering, Tokyo University of Agriculture and Technology, \\ Japan \\ ${ }^{2}$ Ishikawa National College of Technology, Japan \\ ${ }^{3}$ Advanced Industrial Science and Technology, AIST, Japan \\ ${ }^{4}$ Zhejiang Feida Environmental Science \& Technology Co., Ltd., China \\ ${ }^{5}$ Faculty of Physics, University of Vienna, Austria
}

\begin{abstract}
For standardisation of sampling and measuring methods regarding in-stack measurement of PM2.5 and PM10 mass concentration in flue gas of stationary emission sources, a two stage VIS impactor (virtual impaction surface impactor) was developed in which the solid impaction surface of a conventional impactor is replaced by a space of a relatively slow moving air within a cavity of a receiving nozzle. Separation performance of the VIS impactor was examined using a model aerosol-gas stream system with a variety of dust concentrations and then exploited for the flue gas measurement in a coal combustion plant in China. Because a further stack emission is the condensable suspended particle matter (condensable SPM), two types of diluters for this measurement were designed and constructed based on England et al. and W. Lee et al.'s apparatus. Model exhaust gas with heavy metals was prepared in a laboratory scaled experimental arrangement and was mixed with clean air in both dilutor types. The effects of diluter structure, dilution ratio and residence time on the size distribution of condensable SPM are discussed.
\end{abstract}

Keywords: virtual impaction surface impactor, separation efficiency, dilutor, condensable suspended particulate matter. 


\section{Introduction}

PM2.5 (particles which pass through a size-selective nozzle with a 50\% efficiency cut-off at 2,5 $\mu \mathrm{m}$ aerodynamic diameter) invades the endocyst of the lung and is considered to be one of the factors of coronary diseases such as asthma or lung cancer. High correlation of dust concentration and human health was reported [1]. ISO7708 [2] describes the ratios of particles invading the human body by breathing as a function of aerodynamic particle diameter; particles with passage through pharynx (Thoracic convention) (probability of $50 \%$ ) are $10 \mu \mathrm{m}$ and particles reaching alveolus (Respirable convention) are 2.5 $\mu \mathrm{m}$ or smaller and affect particularly the high risk group (sick and infirm, or children). Recently (September 2009), the implementation of Japan environmental standards regarding PM2.5 mass concentration necessitates a standardized measurement of PM2.5 in flue gas from stationary sources. Evidently, the status of PM10 (particles which pass through a size-selective nozzle with a 50\% efficiency cut-off at $10 \mu \mathrm{m}$ aerodynamic diameter)/PM2.5 emission is necessary to investigate. Current status for PM2.5 emission from stationary sources is less recognized comparing that from mobile sources.

In ISO/TC146 "Air quality"/SC1 "Stationary source", working group, WG20, discusses the standard of PM10/PM2.5 mass concentration measurement in stack of stationary sources. ISO 23210 [3], conventional cascade real impactor method, has already been published in 2009. Conventional impactors have collection plates on which particles within a certain size interval are collected following a collision of those particles with the impaction surface. However, particle bounce [4] and re-entrainment [5] occurring particularly at high dust concentration conditions result in substantial errors regarding mass concentrations. When a glass fiber filter was used on a collection plate, John et al. [6] reported that the separation efficiency of a real cascade impactor for coarse particles larger than $2.5 \mu \mathrm{m}$ was about $70 \%$. Based on this result, a permissible deviation of a real impactor from recommended separation efficiency in ISO 7708 was $30 \%$ in published ISO23210. However, the low separation efficiency of coarse particles results in an undesirable overestimation of PM10/PM2.5 mass concentration.

An impactor, in which the solid impaction surface is replaced by a space of a relatively slow moving air within a cavity of a receiving nozzle, was described earlier by Conner [7] and named a virtual impactor. This type of an impactor avoids in general particle bounce and re-entrainment. Since then various modifications and improvement of this type of an impactor were developed as fine particle sampler for environment. A two-stage and multiple-nozzle Virtual Impaction Surface (VIS) impactor [8] has been developed with the aim to serve as a new standard method.

A further category of the emission from stationary sources is the category of condensable SPM. Since dust collectors are generally operated in the range of $100-300^{\circ} \mathrm{C}$ to avoid vapor condensation, the candidates for condensable SPM in exhaust gas were in vapor state when they passed through the dust collectors. After the exhaust gas cooling in the flue gas ducting located downstream of the 
dust collectors mixes with atmospheric air at the exit of the stacks, thereby the condensable components in exhaust gas become particulates. In order to determine the emission behavior of condensable SPM from stationary sources, there is a need to develop a standard method of dilution and its operation conditions, such as the structure of dilutors, dilution rate and residence time in a dilutor. Various types of dilutors were designed and prepared. Number based size distributions of condensable SPM from different stationary sources were measured using each dilutor.

The objective of this study is to establish measurement methods of PM2.5/PM10 and condensable SPM from stationary sources and to accumulate such emission data. Separation efficiency and mass concentration of PM10/PM2.5 in model dust dispersed in an air flow channel were investigated using both types of impactors: the VIS and the conventional cascade impactor.

Furthermore, two types of diluters were designed and constructed based on England et al. [9] and W. Lee et al.'s apparatus [10]. Model exhaust gas with heavy metals was prepared in a laboratory scaled experimental arrangement and was mixed with clean air in both dilutor types. The effects of diluter structure, dilution ratio and residence time on the size distribution of condensable SPM were investigated and by using model exhaust gas and flue gas sample from pulverized coal combustion test facility.

\section{Experimental procedure}

\subsection{PM10/PM2.5 mass concentration measurement by using VIS impactor and conventional (real) cascade impactor}

Two kinds of separation and collection method for the measurement of PM10/PM2.5 mass concentration in stack were used in this study. Each method is in a process of becoming a standard method worked out in ISO TC146/SC1/ WG20.

\subsubsection{VIS impactor}

The principle of operation and the key design parameters of VIS impactor are shown in fig. 1. The particle-laden gas enters the particle acceleration nozzles and accelerates depending on $D_{0}$ and the total flow rate $\mathrm{Q}_{0}$. Only a part of the stream $Q_{1}$ leaving the acceleration nozzles enters the particle collection nozzles. Flow rate through particle collection nozzles, which is called the minor flow rate $\mathrm{Q}_{1}$, is about $10 \%$ of the total flow rate $\mathrm{Q}_{0}$. The remaining, larger part of the flow, the major flow $\mathrm{Q}_{2}$, is redirected and by-passes the particle collection nozzles.

Coarse particles over a certain aerodynamic size (cut-off size) entrained to the minor flow are received by the particle collection nozzles and after passing through those nozzles collected on a filter. Fine particles smaller than this cut-off size stay in the major stream, and are directed into the next separation stage. There are two separation stages (first stage: $10 \mu \mathrm{m}$ cut-off, second stage: $2.5 \mu \mathrm{m}$ cut-off) in the VIS impactor presented in this study. Each stage has 6 acceleration and 6 collection nozzles. Particles with aerodynamic diameters over 
$10 \mu \mathrm{m}$ are sampled on the filter of the first stage, those in the range of 10 to 2.5 $\mu \mathrm{m}$ are sampled on the filter of the second stage and those which belong to the PM2.5 size fraction are sampled on final PM2.5 collection filter.

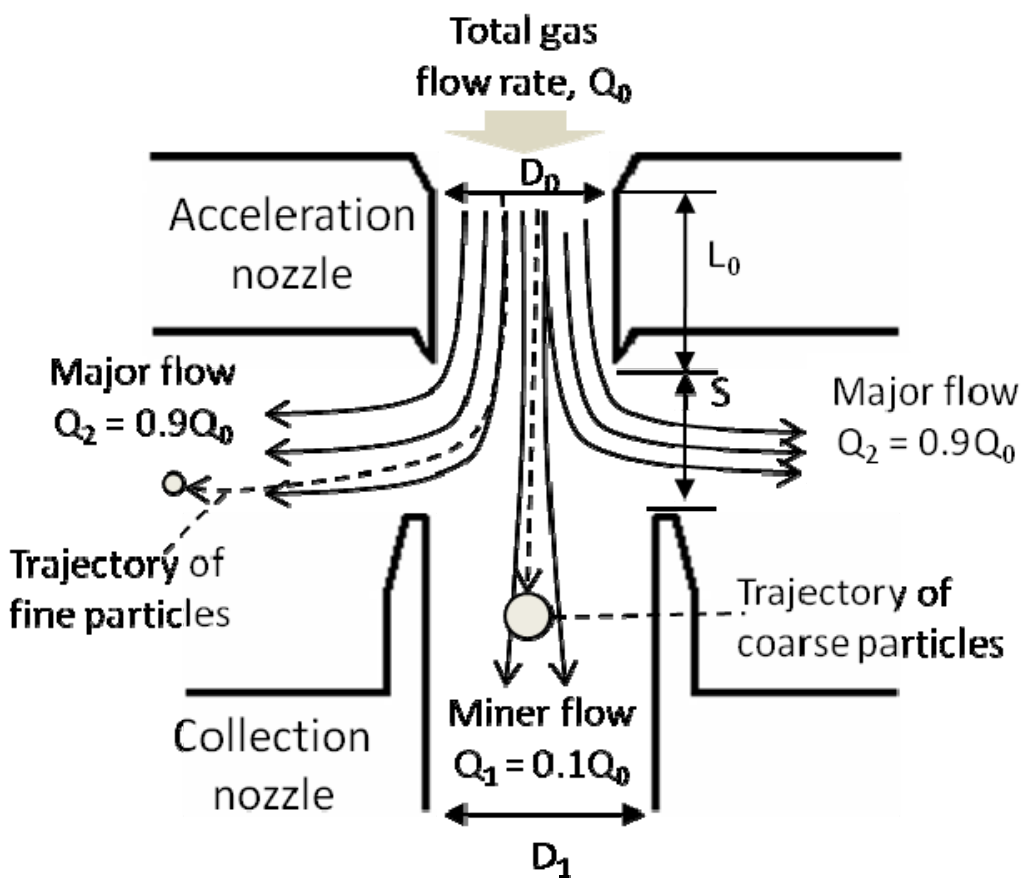

Figure 1: $\quad$ Principle of virtual impaction surface (VIS) impactor.

\subsubsection{Conventional cascade impactor}

A commercially available cascade impactor (GMU-Cascade Impactor Johnas II, Paul Goethe, Bochum, Germany) was tested in comparison with VIS impactors. The GMU impactor has two separation stages (first stage: $10 \mu \mathrm{m}$ cut-off, second stage: $2.5 \mu \mathrm{m}$ cut-off). The first and second stages have 6 and 12 separation nozzles, respectively. In general, coating of impaction plates with grease should limit the particle bounce and re-entrainment at the impaction plates improving the size separating performance of the impactor. However, in stacks at high temperatures in reactive atmospheres, substantial errors in PM10 and PM2.5 mass concentration measurements caused by adhesion degradation (grease) and/or weight change of coated grease are to be worried about. In this study, each impaction stage of GMU impactor with either quartz glass fiber filters or greased metal filters were used. The grease used in these experiments was fluorine corollary grease (Nichimoly, OCE@NFGS Spray). 


\subsubsection{PM2.5 mass concentration measurement using model aerosol generator and stack chamber}

The schematic drawing of model aerosol gas stream duct simulating some industrial conditions is shown in fig. 2. JIS Z8901 Type 1 testing powder \#10 (Fly ash, mass based median aerodynamic particle diameter of $7.9 \mu \mathrm{m}$, geometry standard deviation of $1.2 \mu \mathrm{m}$, particle density of $2000-2300 \mathrm{~kg} / \mathrm{m}^{3}$ ) [11] was used as model dust, and was supplied into model duct chamber by an electric dust feeder. The aggregates of dust were distributed with a mechanical brush. Mass concentration in gas stream was controlled by the feed rate of dust.

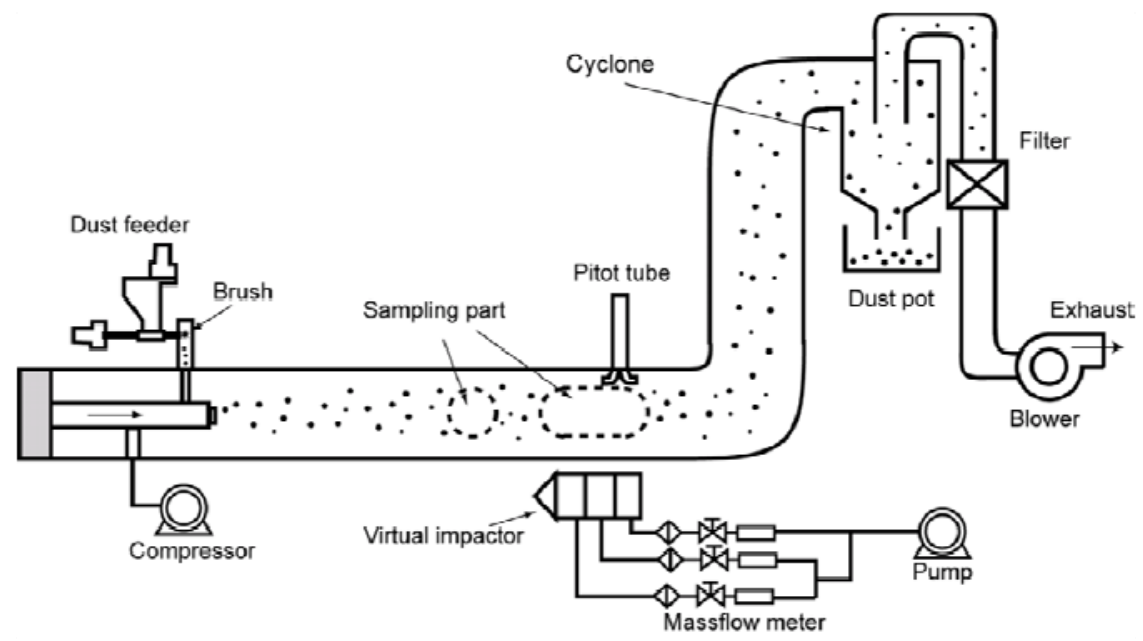

Figure 2: Schematic drawing of model testing chamber.

Since there is possibility that coarse particles in testing powder were settled out by gravity, the size distribution of dispersed model powder was measured by the following method. Dispersed model powders in gas stream were collected sampling circular filter with $37 \mathrm{~mm}$ in diameter at sampling part in dust chamber. The part of collected dust layer on filter was dispersed into pure water under the ultrasonic irradiation. The size distribution of collected dust was measured by a laser diffraction particle size analyzer (Shimadzu Co. Ltd., Japan, SALD-2200). In order to reduce the effect of remained particles in filter after ultrasonic irradiation on size distribution, the amount of dust layer on filter was about $50 \mathrm{mg}$, and dust layer was dispersed into water. Since coarse particles settled down during particle dispersion and transport processes, the amount of fine powder with smaller than $2.5 \mu \mathrm{m}$ in diameter increased from about $7.0 \mathrm{wt} \%$ in original dust to $14 \mathrm{wt} \%$ in collected dust on filter.

After total dust concentration measurement, VIS impactor and/or cascade impactor was installed into model dust chamber, and the effect of total dust concentration and impactor structure on PM2.5/PM10 mass concentration determination was investigated. 


\subsubsection{PM2.5/PM10 mass concentration measurement from coal combustion flue gas in China}

PM10/PM2.5 mass concentration was measured at the test plant of dust collectors in China. The plant combusts coal and a part of flue gas is usually introduced to an electric precipitator (ESP) and bag filters. Sampling point was the center of duct at $8.0 \mathrm{~m}$ downstream of bag filter unit outlet. The gas conditions were: temperature $99^{\circ} \mathrm{C}, \mathrm{CO}_{2}: 8.8 \%, \mathrm{O}_{2}: 10.9 \%$, water content 60 $\mathrm{g} / \mathrm{m}^{3}$, dust concentration $120 \mathrm{mg} / \mathrm{m}^{3}$, and gas velocity $13 \mathrm{~m} / \mathrm{s}$. For our measurements, ESP was stopped and a part of filters in a bag filter unit was removed in order to obtain high dust concentration in the gas. Consequently, PM10/PM2.5 was measured by virtual impactors and cascade real impactor (GMU-impactor) in different, relatively high total dust concentrations.

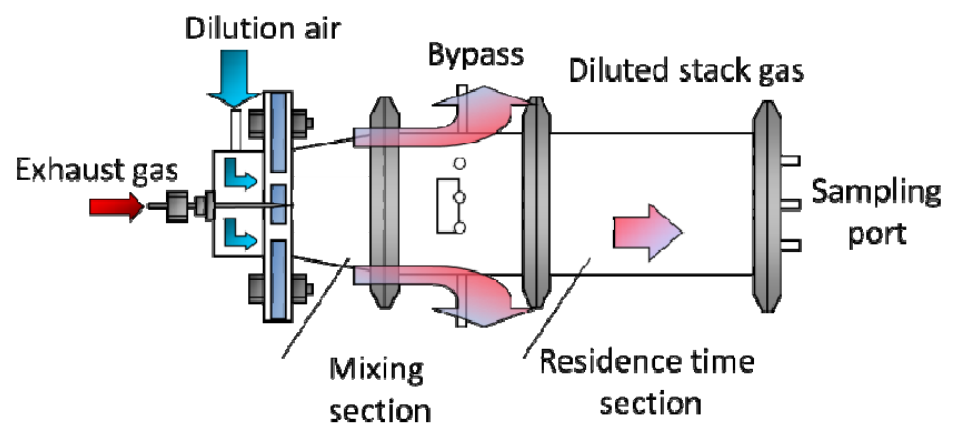

(a) ASTM type dilutor
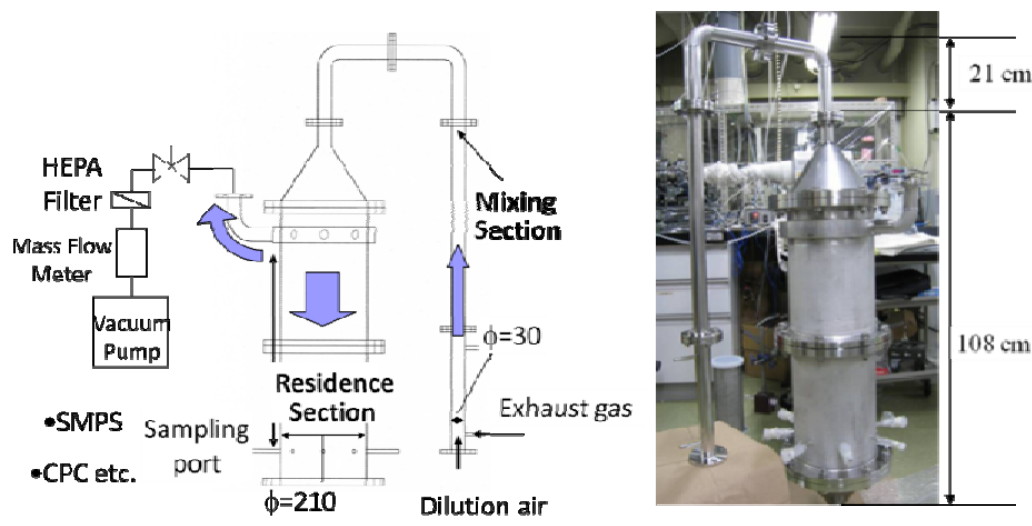

(b) CANMET type dilutor (design drawing and photograph)

Figure 3: Detail structure of two types of dilutors. 


\subsection{Condensable SPM measurement by using dilutors with different designed structure}

\subsubsection{Dilutor}

ASTM [9] based type and CANMET [10] based type dilutors, shown in fig. 3, were designed. Both allow the control of the residence time by adjusting bypass gas flow rate sucked from the jacket near entrance of residence section. Detail of gas suction part was determined based on the flow patters simulated by fluid mechanic software (COMSOL) to ensure homogeneous suction for uniform flow pattern in dilutor.

\subsubsection{Model flue gas with $\mathrm{Cd}$ vapor}

An apparatus for laboratory model flue gas generation and measurement system is shown in fig. 4. $\mathrm{CdCl}_{2}$, which is chosen as an example of source of emission element in waste incineration processes, was sublimed at $450^{\circ} \mathrm{C}$ and carried by dried air, and introduced to a dilutor with dry air dilution.

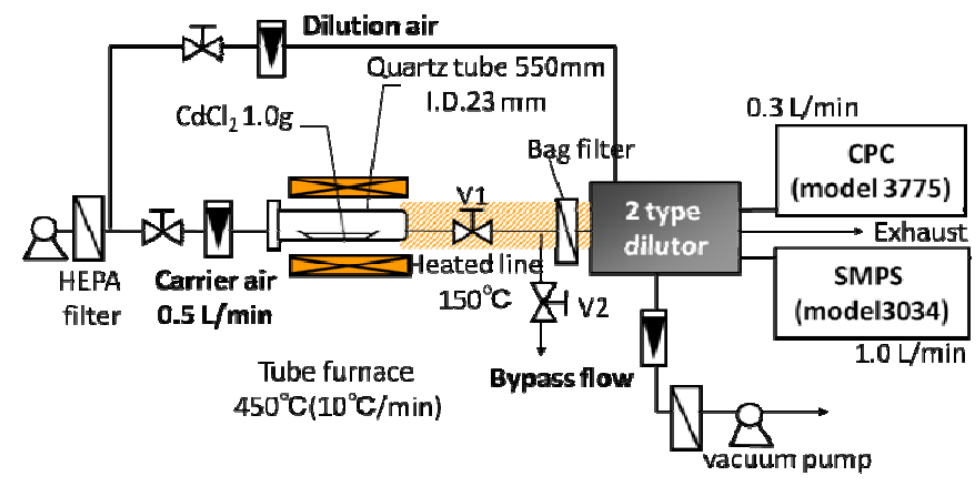

Figure 4: Experimental apparatus for the measurement of condensable SPM using model flue gas with $\mathrm{Cd}$ vapor.

\section{Results and discussion}

\subsection{PM10/PM2.5 mass concentration}

\subsubsection{Model aerosol}

Relationship between the result of measured PM2.5 concentration and the overall dust concentration is shown in fig. 5. The overall dust concentration is calculated by totaling of dust collection at each stage. In case of VIS impactor, PM2.5 concentrations are in a very good agreement with the PM2.5-line that indicates PM2.5 content over the entire dust concentration range.

In case of GMU cascade impactor, even at concentrations lower than 5 $\mathrm{mg} / \mathrm{m}^{3}$, PM2.5-line. Better agreement (GMU) was observed in cases when 
impaction plates were coated with the grease. Though the GMU cascade impactor performance was improved with the grease coating, the values were still higher than those obtained by the VIS impactor.

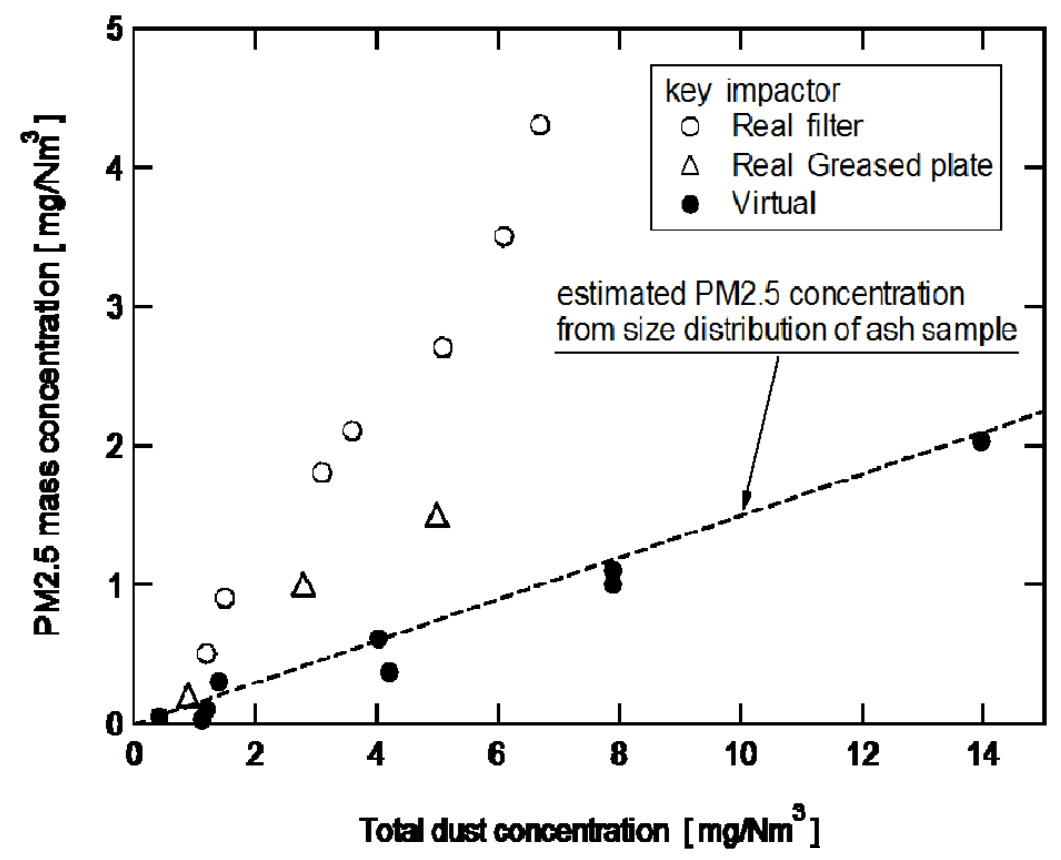

Figure 5: Relationship between total dust concentration and PM2.5 mass concentration measured by using different sampling method.

\subsubsection{Coal combustion flue gas}

The mass concentration of PM10 and PM2.5 was measured by two kinds of impactor in flue gas of coal combustion in China and shown in fig. 6. Since it was difficult to keep stable operation of coal dust collection, it is not enough to discuss the separation performance of both methods. However, it seems that PM2.5 mass concentration measured by GMU cascade real impactor was higher than that by VIS impactor at about almost same total dust concentration ranging less than $100 \mathrm{mg} / \mathrm{Nm}^{3}$.

In order to discuss this difference, SEM images of collected dust at each stage are shown in fig. 7. Particles larger than cut-off diameter were observed which is likely caused by bounce and re-entrainment in the GMU-impactor case. In the virtual impactor case, large particles were also observed, however, the amount of coarse particles larger than $2.5 \mu \mathrm{m}$ was very limited as can also be seen in fig. 7(b). 


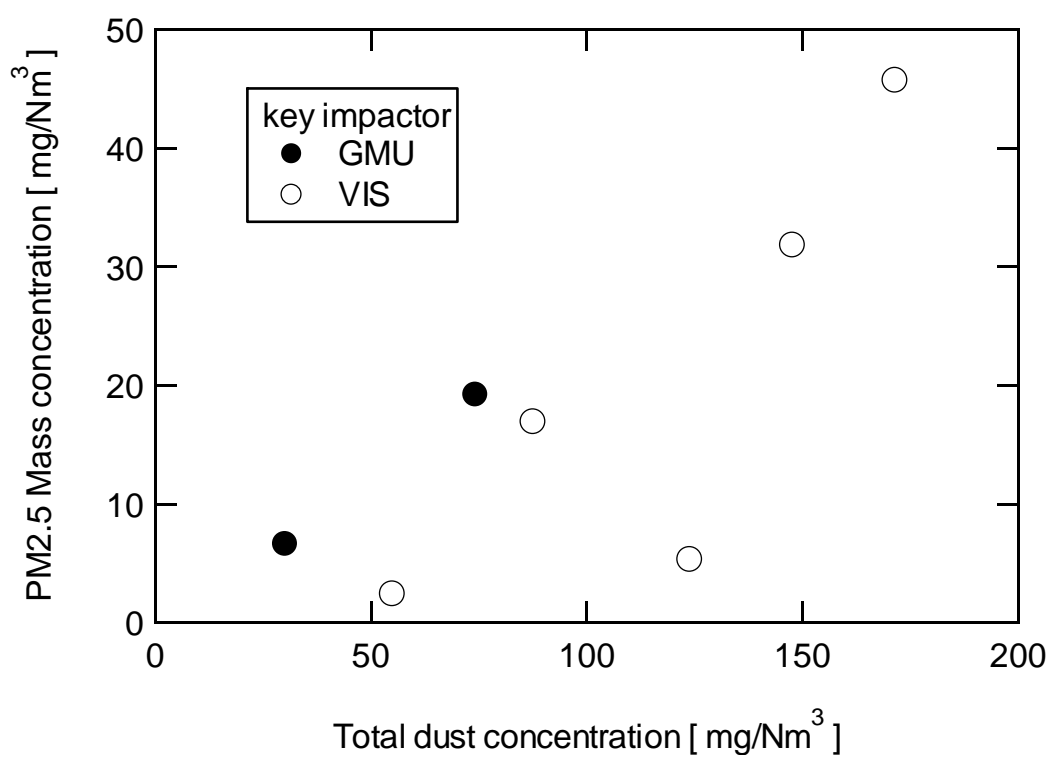

Figure 6: Relationship between total dust concentration and PM2.5 mass concentration in flue gas of coal combustion in China.

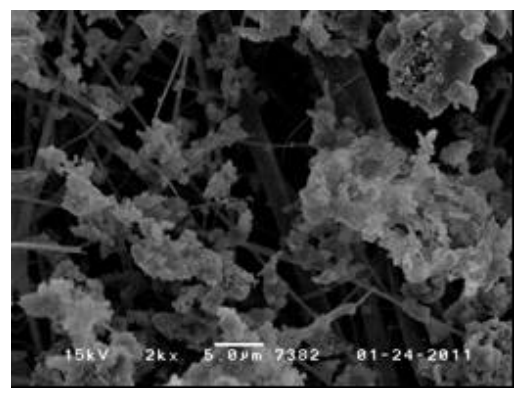

(a) GMU real impactor

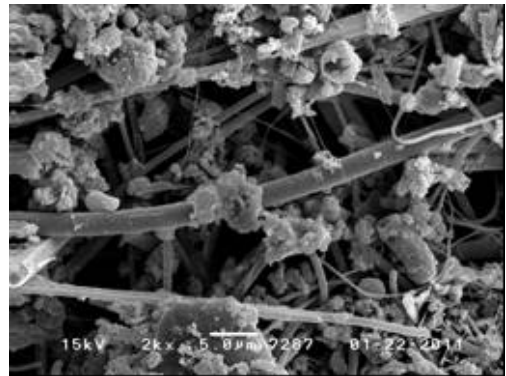

(b) VIS impactor

Figure 7: SEM observation of final PM2.5 stage filter. Effect of separation method of PM2.5 on particle size of PM2.5.

\subsection{Condensable SPM}

The effect of dilution condition on number based size distribution of condensable SPM is shown in fig. 8. Dilution ratio and residence time were changed ranging from 10 to 30 times and from 11 to $52 \mathrm{~s}$, respectively. When dilution ratio was higher than 20 times and residence time was longer than $10 \mathrm{~s}$, almost same size distribution was observed. 


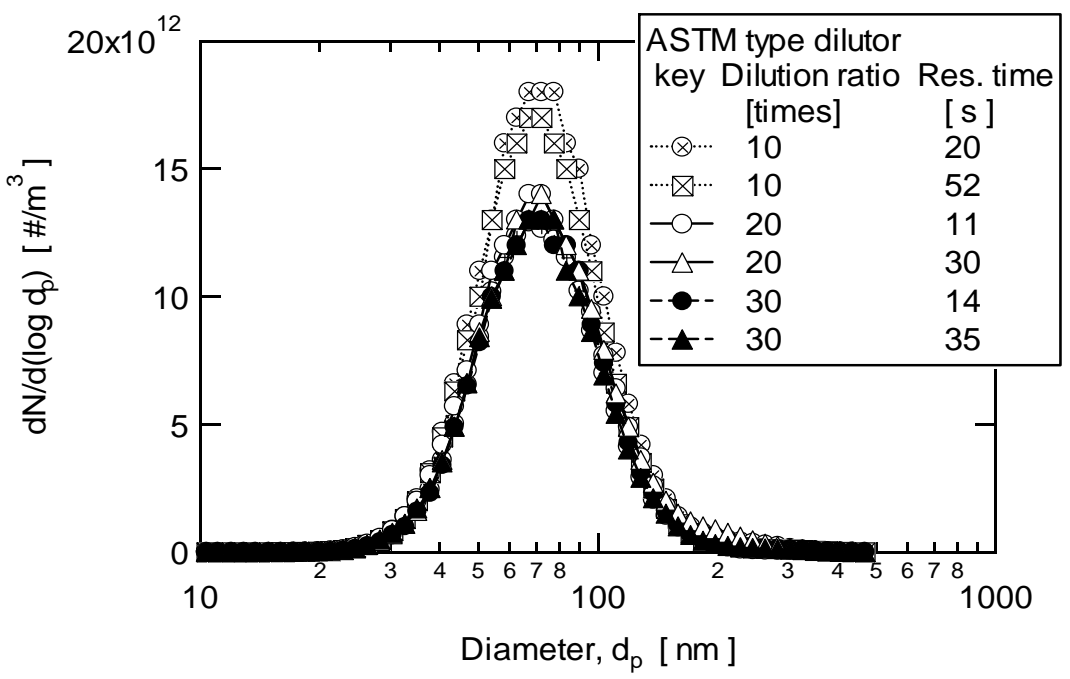

Figure 8: Effect of dilution ratio and residence time on size distribution of condensable SPM. (ASTM type dilutor).

The effect of dilutor structure on number based size distribution of condensable SPM is shown in fig. 9. Almost the same particle size distribution was obtained by using both dilutors. If dilution ratio (DR) was larger than 20 times and residence time (RT) longer than $10 \mathrm{~s}$, fairly well agreements of size distribution are also obtained in two different dilutors.

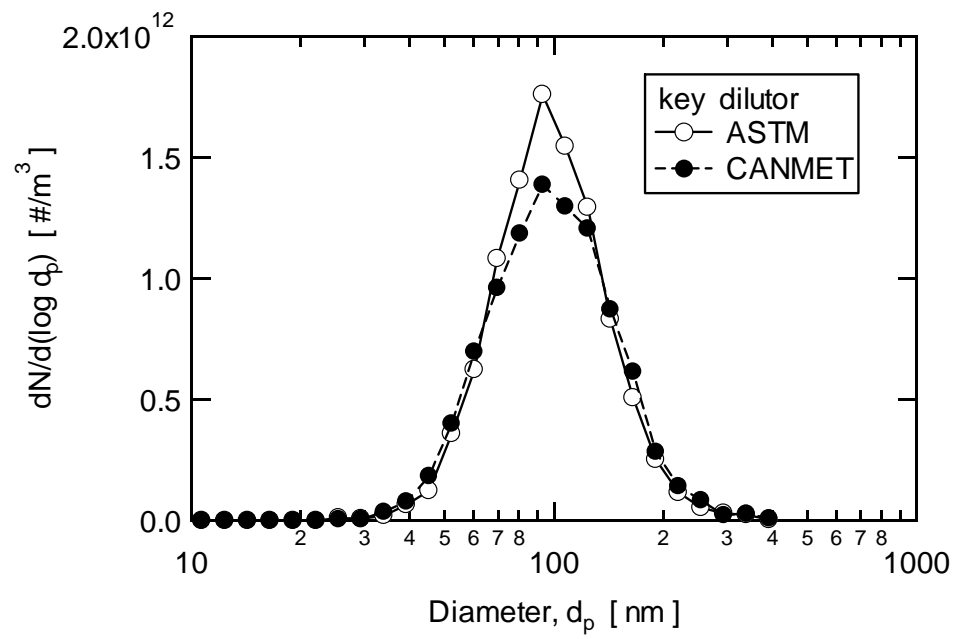

Figure 9: Effect of dilutor structure on size distribution of condensable SPM from model flue gas with $\mathrm{Cd}$ vapor (Dilution ratio : 40 times, Residence time : $55 \mathrm{~s}$ ). 


\section{Conclusion}

The conventional cascade impactor overestimated PM2.5 concentrations due to the particle bounce and re-entrainment even when adhesive coating material such as grease was applied to its impaction plates. VIS impactor showed remarkably good performance within the investigated range of PM2.5 mass concentrations and ambient parameters in the model aerosol gas flow and in the coal combustion flue gas.

With dilution ratio and residence time larger than 20 times and longer than 10 seconds, almost the same number based size distribution of condensable SPM from model flue gas with $\mathrm{Cd}$ vapor using two different types of dilutor was measured.

\section{Acknowledgements}

This work was supported by NEDO Intellectual Infrastructure Program, Research and Development to Promote the Creation and Utilization of an Intellectual Infrastructure (FY2005-2007), NEDO International Standardization Program (FY2008-2010), Grant-in-Aid of JSPS for Young Scientists (B) (20710028) and Grant-in-Aid of MEXT for Scientific Research on Innovative Areas (20120004).

\section{References}

[1] Dockery, D. W., C. A. Pope, III, X. Xu, et al., New England Journal of Medicine, 329, 1753-1759, 1993.

[2] ISO 7708: Air quality — particle size fraction definitions for health-related sampling, 1995.

[3] ISO 23210: Stationary source emissions - Determination of PM10/PM2.5 mass concentration in flue gas - Measurement at low concentrations by use of impactors, 2009.

[4] T. G. Dzubay, L. E. Hines and R. K. Stevens, Atmospheric Environment, 10, 229-234, 1976.

[5] K. Iinoya, S. Yuu, K. Makino and K. Nakano, Kagaku Kogaku, 33, 689698, 1969.

[6] A.C. John, T.A.J. Kuhlbusch, H. Fissan, G. Bröker, K.-J. Geueke, Aerosol Science and Technology 37 694-702, 2003.

[7] W. D. Conner, Journal of the Air Pollution Control Association, 16, 35-38, 1966.

[8] T. Prasserttachato, A. Podgorski, J.H. Luckner, M. Furuuchi, L. Gradon, S. Suvachittanont and W.W. Szymanski, Aerosol and Air Quality Research, 6(1), 67-81, 2006.

[9] G. C. England et al, Journal of the Air and Waste Management Association, 57, 65-78, 2007.

[10] S. Win Lee, T. Herage, I. He and B. Young, Powder Technology, 180(1-2), 145-150, 2008.

[11] JIS Z 8901-1995: Test powders and test particles. 